\title{
Correlation of High PIRADS Score on Three-Tesla Magnetic Resonance with In-Gantry Magnetic Resonance Guided Biopsy in Patients with Clinical Risk of Prostate Cancer
}

\author{
Rajeev Jyoti1,2,3, Hodo Haxhimolla3,4, N. Hamesh Jina ${ }^{4}$ \\ ${ }^{1}$ Department of Radiology, Calvary Hospital, Bruce, ACT, Australia \\ ${ }^{2}$ Universal Medical Imaging Canberra, Calvary Hospital, Bruce, ACT, Australia \\ ${ }^{3}$ Australian National University, Canberra, ACT, Australia \\ ${ }^{4}$ Department of Urology, The Canberra Hospital, Garran, ACT, Australia \\ Email: hameshjina@gmail.com
}

Received 15 December 2015; accepted 25 March 2016; published 29 March 2016

Copyright (C) 2016 by authors and Scientific Research Publishing Inc.

This work is licensed under the Creative Commons Attribution International License (CC BY). http://creativecommons.org/licenses/by/4.0/

(c) (†) Open Access

\section{Abstract}

Introduction and Objective: Prostate cancer detection is a difficult process despite different modalities that are available. The current standard of practice is based on stratifying risk using Prostate Specific Antigen (PSA), digital rectal examination (DRE) and performing a transrectal ultrasound (TRUS) or transperineal (TP) guided biopsy. Recent advances in three-tesla multiparametric magnetic resonance imaging (MP-MRI) technology and the availability of in-gantry MRI guided biopsies (MRGB) have added another diagnostic tool in management of prostate cancer. We reviewed MRGB performed on high score Prostate Imaging Reporting and Data System (PIRADS) score lesions in a single centre retrospective study. Materials and Methods: There were 77 patients (mean age 63) with high PIRADS score (4 and 5) that underwent in-gantry MRGB. All the biopsies were performed utilizing Dyna TRIM prostate biopsy system on a three-tesla MRI scanner by an urologist with assistance of an experienced radiologist. Two to three samples were obtained from each lesion using an MRI compatible 18-gauge biopsy needle. Three experienced pathologists evaluated the samples and provided the results and Gleason score in each positive sample. Results: Out of the total 77 high PIRADS patients, 54 were PIRADS score $4(70 \%)$ and 23 PIRADS score 5 $(30 \%)$. There were 22 positive biopsies for adenocarcinoma of prostate with a Gleason score of $3+$ $3=6$ or higher. Out of the 54 PIRADS score 4 lesions, 13 were positive (24\%) and out of 23 PIRADS 
5 lesions, 9 were positive (39\%). The remaining 55 biopsies were negative for prostate cancer. Conclusion: We present our series of MRGB in patients with a high PIRADS score for prostate cancer. While this diagnostic paradigm was in its infancy stages, MRGB was positive in 24\% of PIRADS 4 and $39 \%$ of PIRADS 5 lesions in this series.

\title{
Keywords
}

\author{
Prostate Cancer, Multiparametric MRI, MP-MRI, Prostate Imaging and Reporting Data System, \\ PIRADS, MRI Guided Biopsies, MRGB
}

\section{Introduction}

Prostate cancer represents 30\% of all male cancer diagnosed and is the second most common cause of cancer death in males in Australia and New Zealand [1] [2], thus representing a serious morbidity and mortality challenge in society. Due to increasing longevity of life, the early diagnosis and treatment of prostate cancer is now more important than ever.

The initial workup of prostate cancer involves stratifying the risk of potential disease using the prostate specific antigen (PSA) blood test and digital rectal examination (DRE). Tissue diagnosis has historically been obtained by transrectal ultrasound (TRUS) or transperineal (TP) sextant biopsies. This currently remains the gold standard of prostate cancer diagnosis [3]. There are, however, limitations with this tool which includes a $21 \%$ false negative rate using the standard 10 - 12 core approach [3] or a 30\% - 45\% risk of cancer up/downstaging with the final pathology [4] [5].

Increasing the number of cores from 12 to 21 increases the detection rate of prostate cancer by $11 \%$ [6]. Even with repeat biopsies, there is an increased probability in making the diagnosis of cancer (an increase of $41 \%$, 31\% and $20 \%$ in detection with three, four or $\geq$ five sets of biopsies respectively), but the morbidity of the additional procedures and delay in diagnosis can be detrimental to the patient [7].

Recent advances and accessibility in multiparametric Magnetic Resonance Imaging (MRI) has significantly enhanced the management options in patients with suspected prostate cancer and improved the accuracy in prostate cancer detection. This has gained popularity as a diagnostic tool in patients who have previously had a negative TRUS biopsy. The Prostate Imaging-Reporting and Data System (PIRADS) score is used in the assessment of suspicious lesions on MRI which is based on structured radiological parameters outlined in the 2012 guidelines from the European Society of Urogenital Radiology [8]. The overall score gives an indictaion of the likelihood of presence of clinically significant cancer within the prostate. The PIRADS system is being adopted gradually in many centres for reporting MRI findings in patients with suspected prostate cancer.

Our centre, which is equipped with a dedicated in-gantry 3.0 Tesla MR guided prostate biopsy system, allowed us to regularly perform these procedures. There is a good body of evidence in current literature demonstrating high accuracy and reliability of in-gantry MR guided prostate biopsy procedure [9]. Prostate cancer detection rates using this technology range from 37\% - 59\% with 93\% of clinically significant disease being detected [10]-[12].

\section{Methods}

We present the results from a single surgeon-radiologist team working in a tertiary hospital that performed MRI guided biopsies over a 15-month period from December 2012. This is a retrospective study and approved by the state ethics committee. Patients recruited in the study were deemed high risk and the inclusion criteria is patients over 50 with an elevated PSA (>2.5) on more than one consecutive reading, a palpable nodule on rectal examination (PR) or ultrasound (US), a previous negative TRUS biopsy and/or a positive family history of prostate cancer. The TRUS biopsies were performed transrectally with 36 core saturation biopsies irrespective of prostate cancer size on TRUS. Patients were excluded from the study if they were known to have prostate cancer and the scan was indicated for surveillance or had an indeterminate or low (PIRADS1-2) score. All patients were given antibiotics perioperatively using the same protocol as for a TRUS biopsy at our centre.

At our institution, all MRI imaging and biopsies were performed using a standard protocol and guidelines. A 
standardized MRGB procedure was performed on each patient, using DYNATRIM (In vivo) needle guide system with $18 \mathrm{G}$ titanium core biopsy needles. 2 - 3 cores were obtained from each lesion. This was performed with the patient in the prone position and utilizing local anaesthetic gel. The minimum lesion size for biopsy was $\mathrm{x} \mathrm{mm}$, and therefore every lesion that was identified was reviewed for biopsy. There was no fusion of the MRI images with an ultrasound scan.

Pre-biopsy prostate MRI was interpreted by a single experienced genitourinary MRI radiologist and the biopsies were interpreted by 2 - 3 pathologists using a standard format. A single MRI report was generated which was standardised and scored as per a modified PIRADS criteria. Dyna TRIM (In vivo) software was utilized for image analysis and generating Apparent Diffusion Coefficient (ADC) values and dynamic perfusion maps. A PIRADS score of 4 or 5 suggested that a clinically significant cancer is likely to be present and thus, the patient proceeded to biopsy. Irrespective of the PIRADS score, all patients were offered the option of a TRUS or MRGB to be performed by the urologist. The first core of MRI guided biopsy was obtained from the centre of an lesion and additional cores were obtained from the areas adjacent to the central core, to minimise sampling error. The repeat in-gantry MRGB was performed on average two months after the initial MRI.

\section{Results}

We recruited 191 patients who met the criteria for high risk and had an MRI prostate performed. The mean age of the patients was 63 with an overwhelming majority Caucasian background. The average size of the gland biopsied on MRI was 73cc (17-263cc range) with a median PSA of $6.37 \mathrm{ng} / \mathrm{ml}$ (0.91 - $15.9 \mathrm{ng} / \mathrm{ml} \mathrm{range).}$

A total of 121 patients out of 191 patients (63\%) received a high PIRADS score, with 75 patients with a PIRADS score of 4 and 46 patients with a PIRADS score of 5 . There were 38 patients in the PIRADS 4 group (51\%) who had a previous negative TRUS biopsy prior to MRGB and 18 in the PIRADS 5 group (39\%).

There were 54 patients (44\%) who underwent MRGB comprising of a total of 77 lesions - 54 PIRADS 4 (70\%) and 23 PIRADS 5 lesions (30\%).

A cancer diagnosis was made with a minimum Gleason score of $3+3=6$. There were $22(30 \%)$ lesionswith cancer detected on the histology, out of which 13 were PIRADS 4 (24\% positive biopsy rate) and 9 were PIRADS 5 (39\% positive biopsy rate) lesions. A limitation of the study is that the individual Gleason scores for the each of the positive biopsies were not correlated with the respective PIRADS scores. This is because the sample size was too small to make any correlation between the Gleason score and PIRADS score and therefore sample bias would be significant. This would mean that the data would not be able to be generalized.

Figures 1-4 demonstrate four different patients who have a PIRADS 5 lesion which returned positive for adenocarcinoma. Figures 1-3 demonstrate the different parameters of the mp-MRI-T2 weighted image, ap-

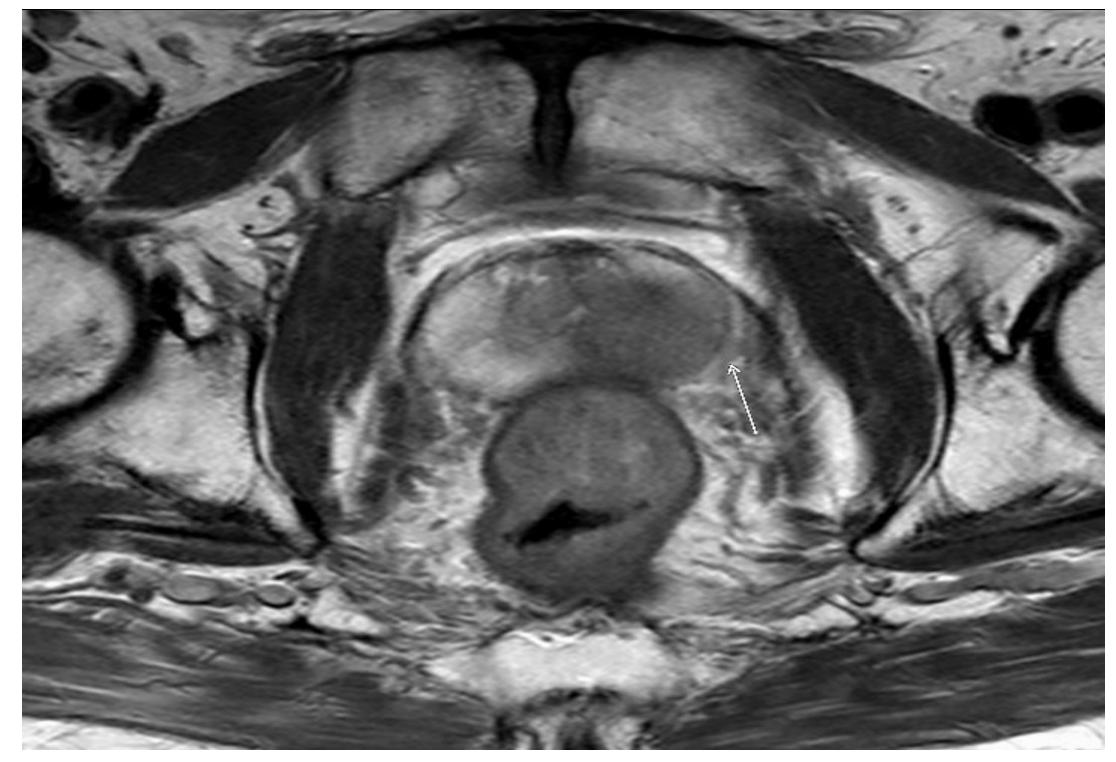

Figure 1. T2 image with a PIRADS five lesion. 


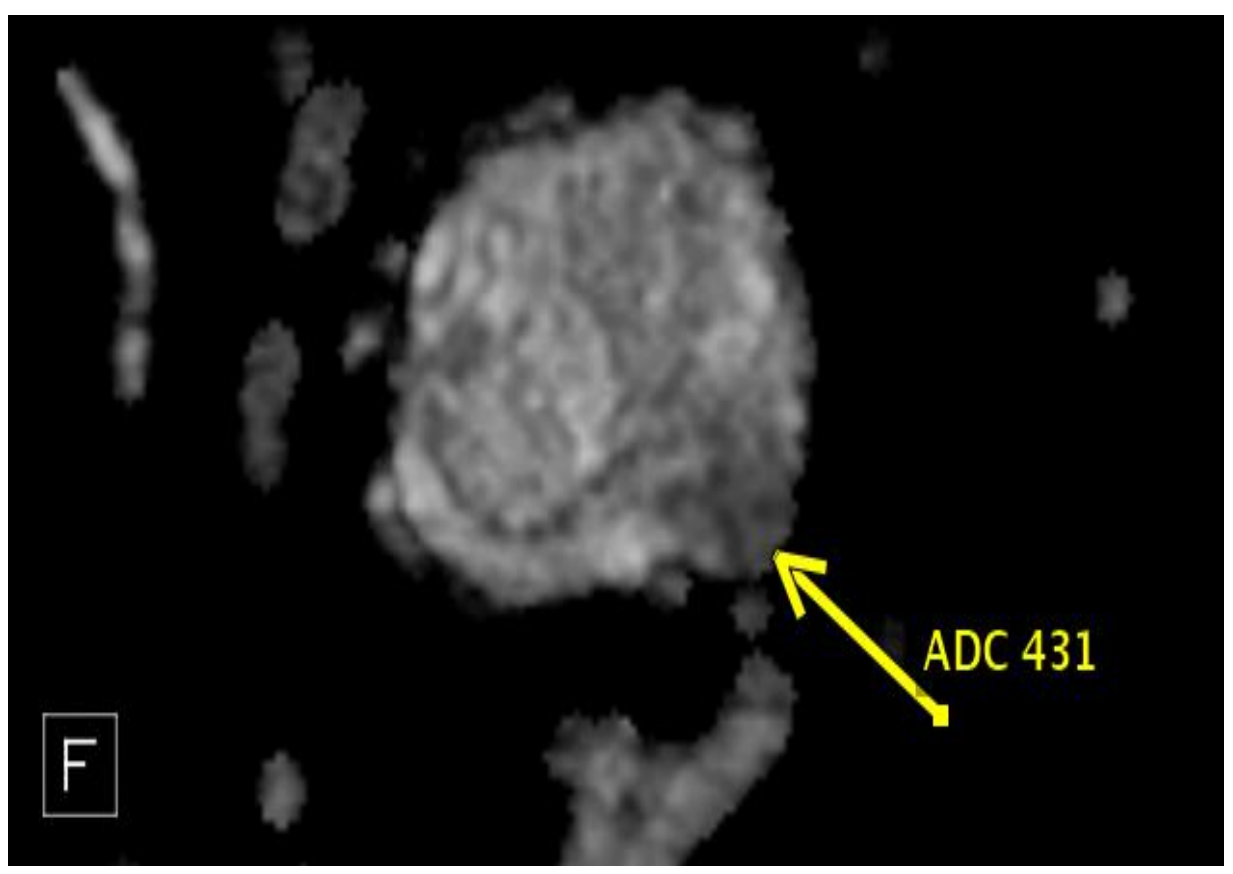

Figure 2. Low ADC in a PIRADS five lesion.

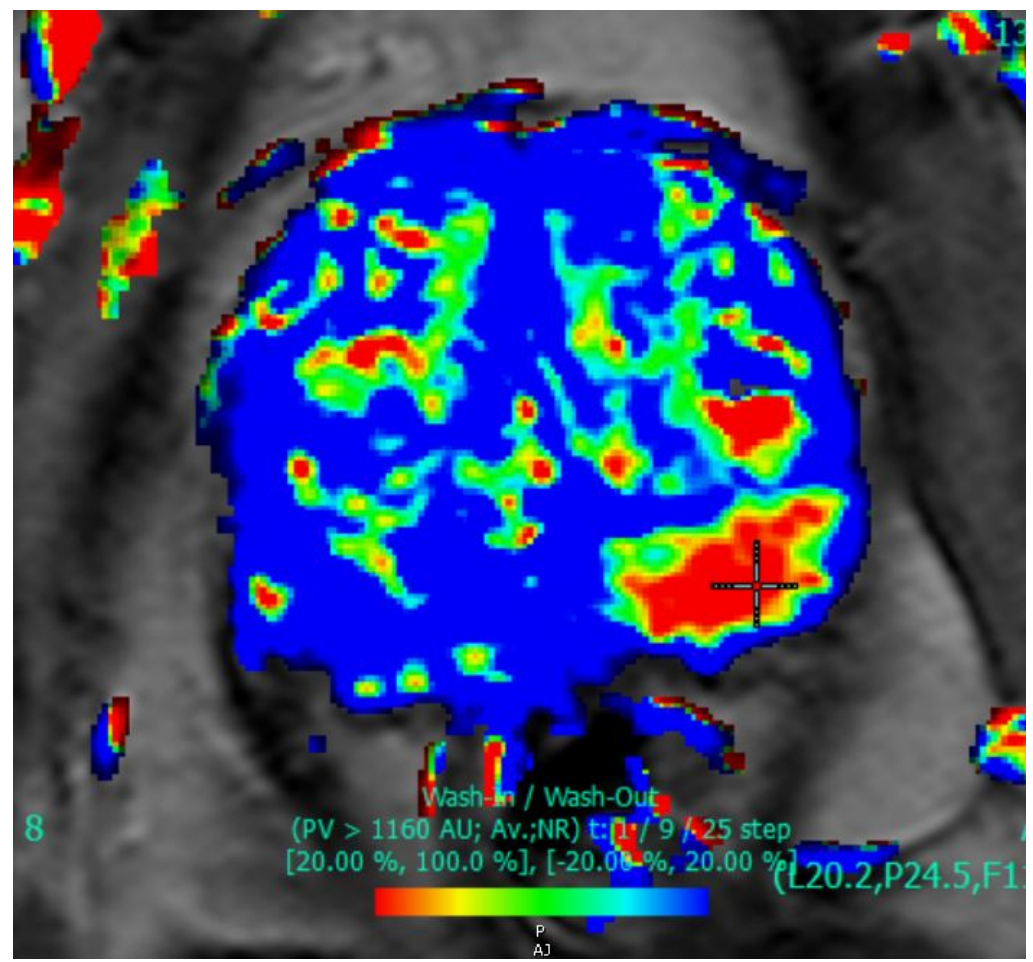

Figure 3. DCE in a PIRADS five lesion.

parent diffusion coefficient (ADC) and dynamic contrast enhancement (DCE). Figure 4 demonstrates the MRGB screen capture.

There were 55 lesions in the high PIRADS 4+ group who had negative biopsies comprising of 41 PIRADS 4 (76\% negative biopsy) and 14 PIRADS 5 (61\% negative biopsy). Of this seven patients had chronic prostatitis on their biopsies. There were no perioperative complications. 


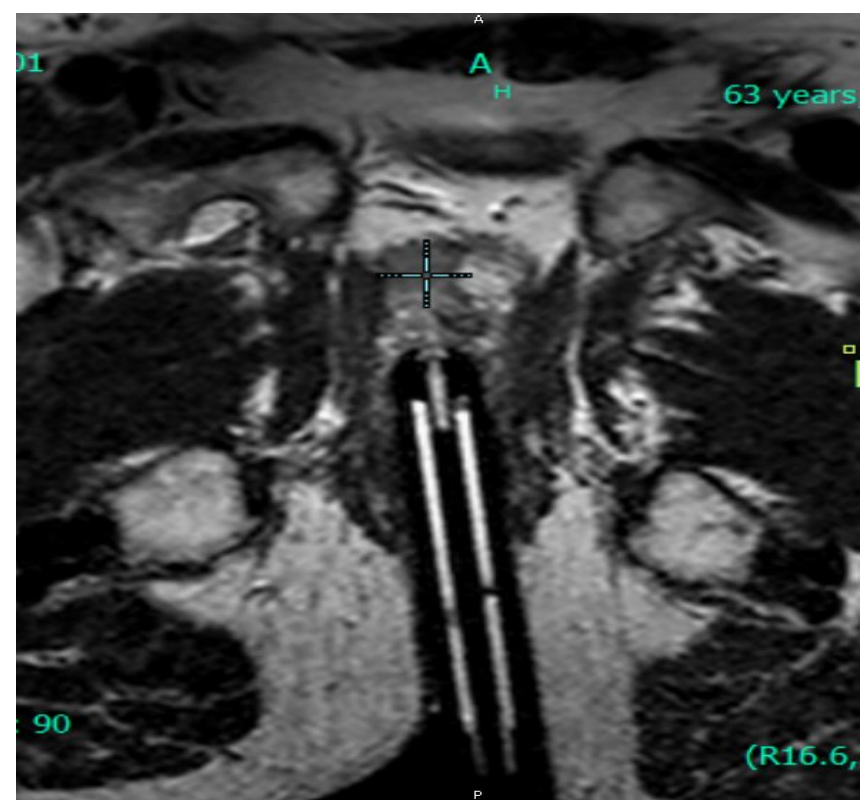

Figure 4. In-gantry MRI guided prostate biopsy.

\section{Conclusions}

MRI of the prostate is increasingly becoming popular as a diagnostic tool for the detection of prostate cancer in patients who have had negative TRUS biopsies, a persistently elevated PSA or a prostate nodule clinically or on US. In some centres now, it is used as a first line tool prior to the initial TRUS or TP biopsy to facilitate targeted biopsies.

We present our series from a single centre evaluating the role of MRGB based on high PIRADS lesions. Our results support a role for MRGB, although our positive biopsy rate of 24\% with PIRADS 4 lesions and 39\% with PIRADS 5 lesions are lower than that of other studies (52\% - 59\% [13]). Only 44\% of patients with high PIRADS score in our series underwent MRGB. Prior studies have shown that MRGB, when compared to a matched reference group who underwent repeated TRUS-biopsy, detected significantly more tumours than standard repeated TRUS-biopsy (22\% for second and 15\% for third TRUS-biopsy[12] [13]). This argument is a strong proponent for MRGB.

We have detected clinically significant disease and other studies have shown that MRI reduces the need for biopsies by $51 \%$ and increases the detection of intermediate/high grade cancer by $17.7 \%$ [14]. This further supports the role of MRGB. Limitations of In-Gantry MRGB include the MRI time required for the procedure (average 40 minutes per patient in our centre), cost of MRI compatible needles and overall cost of the procedure. These factors are likely to be improved with a wider use of this technique and certainly the duration of the procedure decreased over time in our centre. Although not yet widely available, we believe that this technique will be utilized more widely as scanning time and equipment cost improves.

\section{Acknowledgements}

We thank Mr. Jeffrey Wheeler, Universal Medical Imaging, Canberra for manuscript and images preparation.

\section{References}

[1] Australian Institute of Health and Welfare (2012) Cancer Incidence Projections: Australia, 2011 to 2020. Cancer Series No. 66, Cat. No. CAN 62. AIHW, Canberra.

[2] Australian Institute of Health and Welfare (2012) Cancer in Australia: An Overview, 2012. Cancer Series No. 74, Cat. No. CAN 70, AIHW, Canberra.

[3] Noguchi, M., Stameyohn, T.A., McNeal, E. and Yemoto, C.M. (2001) Relationship between Systematic Biopsies and Histological Features of 222 Radical Prostatectomy Specimens: Lack of Prediction of Tumour Significance for Men 
with Nonpalpable Prostate Cancer. Jouranl of Urology, 166, 104-110. http://dx.doi.org/10.1016/S0022-5347(05)66086-7

[4] King, C.R., McNeal, J.E., Gill, H. and Presti Jr., J.C. (2004) Extended Prostate Biopsy Scheme Improves Reliability of Gleason Grading Implications for Radiotherapy Patients. International Journal of Radiation Oncology, Biology, Physics, 59, 386-391. http://dx.doi.org/10.1016/j.ijrobp.2003.10.014

[5] Donohue, J.F., Bianco Jr., F.J., Kuroiwa, K., Vickers, A.J., Wheeler, T.M., Scardino, P.T., et al. (2006) Poorly Differentiate Prostate Cancer Treated with Radical Prostatectomy: Long-Term Outcome and Incidence of Pathological Downgrading. Jouranl of Urology, 176, 991-995. http://dx.doi.org/10.1016/j.juro.2006.04.048

[6] Bjurlin, M.A., Wysock, J.S. and Taneja, S.S. (2014) Optimisation of Prostate Biopsy: Review of Technique and Complications. Urologic Clinics of North America, 41, 299-313.

[7] Stewart, C.S., Leibovich, B.C., Weaver, A.L. and Lieber, M.M. (2001) Prostate Cancer Diagnosis Using a Saturation Needle Biopsy Technique after Previous Negative Sextant Biopsies. Jouranl of Urology, 166, 86-92. http://dx.doi.org/10.1016/S0022-5347(05)66083-1

[8] Barantz, J., Richenberg, J., Clements, R., Choyke, P., Verma, S., Villeirs, G., et al. (2012) ESUR Prostate MR Guidelines 2012. European Radiology, 22, 746-757. http://dx.doi.org/10.1007/s00330-011-2377-y

[9] Hoeks, C.M.A., Schouten, M.G., Bomers, J.G.R., Hoogendoorn, S.P., Hulsbergen-van de Kaa, C.A., Hambrock, T., et al. (2012) Three-Tesla Magnetic Resonance-Guided Prostate Biopsy in Men with Increased Prostate-Specific Antigen and Repeated, Negative, Random, Systematic, Transrectal Ultrasound Biopsies: Detection of Clinically Significant Prostate Cancers. European Urology, 62, 902-909. http://dx.doi.org/10.1016/j.eururo.2012.01.047

[10] Hambrock, T., Somford, D.M., Hoeks, C., Bouwense, S.A., Huisman, H., Yakar, D., et al. (2012) Magnetic Resonance Imaging Guided Prostate Biopsy in Men with Repeat Negative Biopsies and Increased Prostate Specific Antigen. Jouranl of Urology, 183, 520-527.

[11] Roethke, M., Anastasiadis, A.G., Lichy, M., Werner, M., Wagner, P., Kruck, S., et al. (2012) MRI-Guided Prostate Biopsy Detects Clinically Significant Cancer: Analysis of a Cohort of 100 Patients after Previous Negative TRUS Biopsy. World Journal of Urology, 30, 212-218.

[12] Franiel, T., Stephan, C., Erbersdobler, A., Dietz, E., Maxeiner, A., Hell, N., et al. (2011) Areas Suspicious for Prostate Cancer: MR-Guided Biopsy in Patients with at Least One Transrectal US-Guided Biopsy with a Negative FindingMultiparametric MR Imaging for Detection and Biopsy Planning. Radiology, 259, 162-172. http://dx.doi.org/10.1148/radiol.10101251

[13] Hambrock, T., Somford, D.M., Hoeks, C., Bouwense, S.A., Huisman, H., Yakar, D., et al. (2010) Magnetic Resonance Imaging Guided Prostate Biopsy in Men with Repeat Negative Biopsies and Increased Prostate Specific Antigen. Journal of Urology, 183, 520-527. http://dx.doi.org/10.1016/j.juro.2009.10.022

[14] Pokorny, M.R., de Rooij, M., Duncan, E., Schröder, F.H., Parkinson, R., Barentsz, J.O. and Thompson, L.C. (2014) Prospective Study of Diagnostic Accuracy Comparing Prostate Cancer Detection by Transrectal Ultrasound-Guided Biopsy vs. Magnetic Resonance (MR) Imaging with Subsequent MR-Guided Biopsy in Men without Previous Prostate Biopsies. European Urology, 66, 1 22-29. 